\title{
Avaliação discente e as Diretrizes Curriculares Nacionais - realidade das clínicas integradas da UNIVILLE
}

\author{
Lúcia Helena Ferretti*, Tiago Goulart Appel*, Luiz Carlos Machado Miguel**, \\ Luciano Madeira***
}

\author{
* Cirurgiões-dentistas, egressos do Curso de Graduação em \\ Odontologia da Universidade da Região de Joinville - UNIVILLE \\ ** Doutor, Professor da Disciplina de Dentística I e Coordenador \\ do Departamento de Odontologia da Universidade da Região de \\ Joinville - UNIVILLE \\ *** Mestre, Coordenador e Professor da Clínica Integrada Adulta da \\ Universidade da Região de Joinville - UNIVILLE
}

\section{RESUMO}

Após a publicação das DCN os cursos de Odontologia necessitaram se adaptar em suas matrizes curriculares. Esta adaptação envolve não somente uma alteração curricular, mas também incorpora uma nova filosofia pedagógica que procura aproximar os preceitos contidos nas DCN com a prática do dia a dia do ensino nas clínicas integradas dos cursos de Odontologia. A proposta deste trabalho foi analisar a produtividade do aluno de graduação de Odontologia da UNIVILLE nas disciplinas de atividades clínicas, ao longo dos três anos de graduação em cinco (5) diferentes especialidades odontológicas (periodontia, dentística, endodontia, prótese e cirurgia). Foi avaliada a produtividade de oito (8) alunos egressos, quantificando-se sua produção durante os três (3) anos da disciplina de atividades clínicas, $3^{\circ}, 4^{\circ}$ e $5^{\circ}$ anos, especificamente clínicas de baixa, média e alta complexidade. A análise dos dados demonstrou: em endodontia, uma produção média de 4,1 tratamentos endodônticos por aluno; em prótese, uma média de 1,6 próteses fixas unitárias e 1,5 próteses totais por aluno; e, em dentística, uma média de 28 procedimentos restauradores diretos. Com base nos resultados deste modelo de avaliação foi possível concluir que o desenvolvimento de atividades clínicas integradas e por níveis de complexidade permite que o egresso de odontologia realize um aprendizado pedagógico efetivo, com o acompanhamento do paciente em todas as suas fases de diagnóstico, planejamento e conclusão do tratamento. No entanto a pesquisa demonstra que este egresso conclui sua for- mação sem a realização de determinados procedimentos técnicos específicos às especialidades odontológicas pesquisadas.

\section{DESCRITORES}

Clínica Integrada. Produtividade. Diretrizes Curriculares Nacionais.

$\Delta$ tualmente, os Cursos de Graduação em Odontologia têm se preocupado em adequar seus currículos às Diretrizes Curriculares Nacionais, que preconizam que o perfil do egresso deva ser generalista, humanista, crítico e reflexivo, competente técnico e cientificamente, respeitando os princípios legais, éticos e compreendendo a realidade em que se encontra. ${ }^{16}$

Essa proposta contrasta com o modelo de ensino Odontológico que tradicionalmente norteava a formação dos profissionais no Brasil. Este modelo atualmente encontra-se em transição, com uma nova proposta de visão integral do paciente, onde o tratamento exige que o aluno assuma uma postura não somente intervencionista, mas filosófica em relação à saúde integral do paciente. O antigo modelo, denominado "Odontologia Científica ou Flexneriana”, onde o foco é a universalidade biológica, estava orientado para cura ou alívio das doenças ou restauração das lesões. Nos currículos tradicionais dos cursos de graduação em odontologia o atendimento aos pacientes é fragmentado desde o início por clínicas de especialidades, dentre as quais: dentística, prótese, cirurgia, periodontia, endodontia, bem como a 
própria clínica integrada. Assim, nesse modelo de desenvolvimento da atividade clínica, o planejamento e tratamento dos pacientes são voltados para a própria disciplina, não observando o paciente como um todo em sua integralidade.

As atividades clínicas do curso de odontologia da UNIVILLE foram idealizadas sob as Diretrizes do Conselho Nacional de Educação, propondo o ensino de uma visão generalizada do diagnóstico, planejamento e tratamento do paciente, contrapondo à visão fragmentada da odontologia, conforme o modelo tradicional de ensino odontológico. Desta forma, os alunos desenvolvem as clínicas de atendimento, desde o início da prática com pacientes, na forma de "clínica integrada" e não por clínicas de especialidades. De forma a promover um ensino sequencial, respeitando a evolução técnica e científica (teórica) dos alunos, as clínicas foram desenvolvidas por níveis de complexidade (baixa, média e alta), dentro de uma filosofia de promoção de saúde que prioriza o diagnóstico, prevenção e planejamento.

Uma preocupação nesse sistema de desenvolvimento das atividades clínicas é com a produção dos alunos ao longo da formação acadêmica. Nesse modelo de projeto político-pedagógico, que respeita o paciente como um ser integral e prioriza a abordagem generalizada de diagnóstico, planejamento e tratamento dos pacientes, a produtividade não é o foco da disciplina. Enquanto que no currículo tradicional, as clínicas por especialidades preconizavam e exigiam produção mínima para a formação do aluno, no sistema de desenvolvimento da disciplina de atividades clínicas do curso de graduação em odontologia UNIVILLE isso não ocorre, pois no modelo de atendimento integral do paciente não há o estabelecimento de uma produção mínima a ser alcançada.

Nesta nova visão pedagógica, a produtividade do acadêmico poderia ficar comprometida no âmbito especializado, significando que o acadêmico poderia concluir sua formação, sem a realização de procedimentos específicos dentro das especialidades odontológicas, importantes no aprendizado prático do graduando de odontologia.

Inúmeros autores têm se preocupado em estudar o desenvolvimento da disciplina de clínica integrada em diferentes âmbitos como a importância da disciplina na formação do acadêmico, ${ }^{9,2,6,3,13}$ a questão da promoção de saúde nas clínicas de atendimento; ${ }^{1}$ questões discentes e docentes, ${ }^{14}$ bem como a questão da produtividade na clínica integrada ${ }^{8,18}$ e fatores que interferem nessa produtividade. ${ }^{4}$

$\mathrm{Na}$ falta de informações sobre o desenvolvimento da clínica integrada nesse novo modelo pedagógico adequado às novas Diretrizes Curriculares, o objetivo desse estudo foi avaliar a produtividade específica do aluno de graduação, nas especialidades de periodontia, endodontia, dentística, prótese e cirurgia ao longo de sua formação na disciplina de atividades clínicas do Curso de Graduação em Odontologia da UNIVILLE.

\section{MATERIAIS E MÉTODOS}

A metodologia desta pesquisa, aprovada conforme parecer $n^{\circ}$ 163/09 do Comitê de Ética em Pesquisa da UNIVILLE, consistiu em avaliar a produtividade de oito (8) alunos egressos do curso de graduação em odontologia da UNIVILLE, através de consentimento livre e esclarecido. Foi avaliada a produção durante os 3 anos das disciplinas de atividades clínicas $\left(3^{\circ}, 4^{\circ}\right.$ e $5^{\circ}$ anos, especificamente clínicas de baixa, média e alta complexidade), conforme classificação determinada pelo projeto político pedagógico do curso.

Junto ao setor de triagem foi realizado um levantamento dos pacientes atendidos pelos alunos participantes do estudo durante os três anos da disciplina de Atividades Clínicas. Após a determinação dos procedimentos a serem quantificados nas especialidades de endodontia, dentística, periodontia, cirurgia e prótese, foi elaborada uma planilha para registro dos dados e a produção dos alunos participantes foi levantada com base nos registros realizados nos prontuários de cada paciente atendido pelos alunos nesse período.

Após a coleta dos dados, os mesmos foram apresentados em gráficos, por número de produção individualmente e por ano de clínica. Optou-se, neste estudo, pela análise simples dos resultados produzidos uma vez que não era objetivo do trabalho uma comparação entre os egressos participantes do estudo ou comparação com outros modelos de clínica.

\section{RESULTADOS}

Na planilha de coleta de dados houve uma preocupação em registrar a maior quantidade de informações relacionadas aos atendimentos realizados pelos alunos que aceitaram participar da pesquisa. Para cada aluno foi registrado uma série de dados gerais como a quantidade de pacientes atendidos, falta de pacientes às consultas, quantidade de consultas de urgência, a quantidade de consultas para 
realização de plano de tratamento, bem como os procedimentos das diferentes especialidades odontológicas. Nesse trabalho limitamos os dados aos procedimentos relacionados às especialidades de periodontia, endodontia, dentística, cirurgia e prótese, conforme segue:

$\mathrm{Na}$ área de periodontia, devido à variedade de formas de registro nos prontuários dos pacientes, não foi possível a coleta dos dados por procedimentos específicos. Desta maneira todos os procedimentos como raspagem supra e subgengival, profilaxia e remoção de fatores de retenção de placa foram registrados como procedimentos periodontais (Gráfico 1$)$.

Nos Gráficos 2 e 3 pode-se observar a produção desenvolvida pelos alunos na área de endodontia nos três anos de atividades clínicas. A análise dos dados (Gráfico 2) demonstrou uma produção média dos alunos de 1,4 endodontias de dentes uniradiculares e 1,0 dente biradicular. Destaca-se que 37,5\% dos participantes não realizaram nenhuma endodontia de dentes uniradiculares e outros $37,5 \%$ não
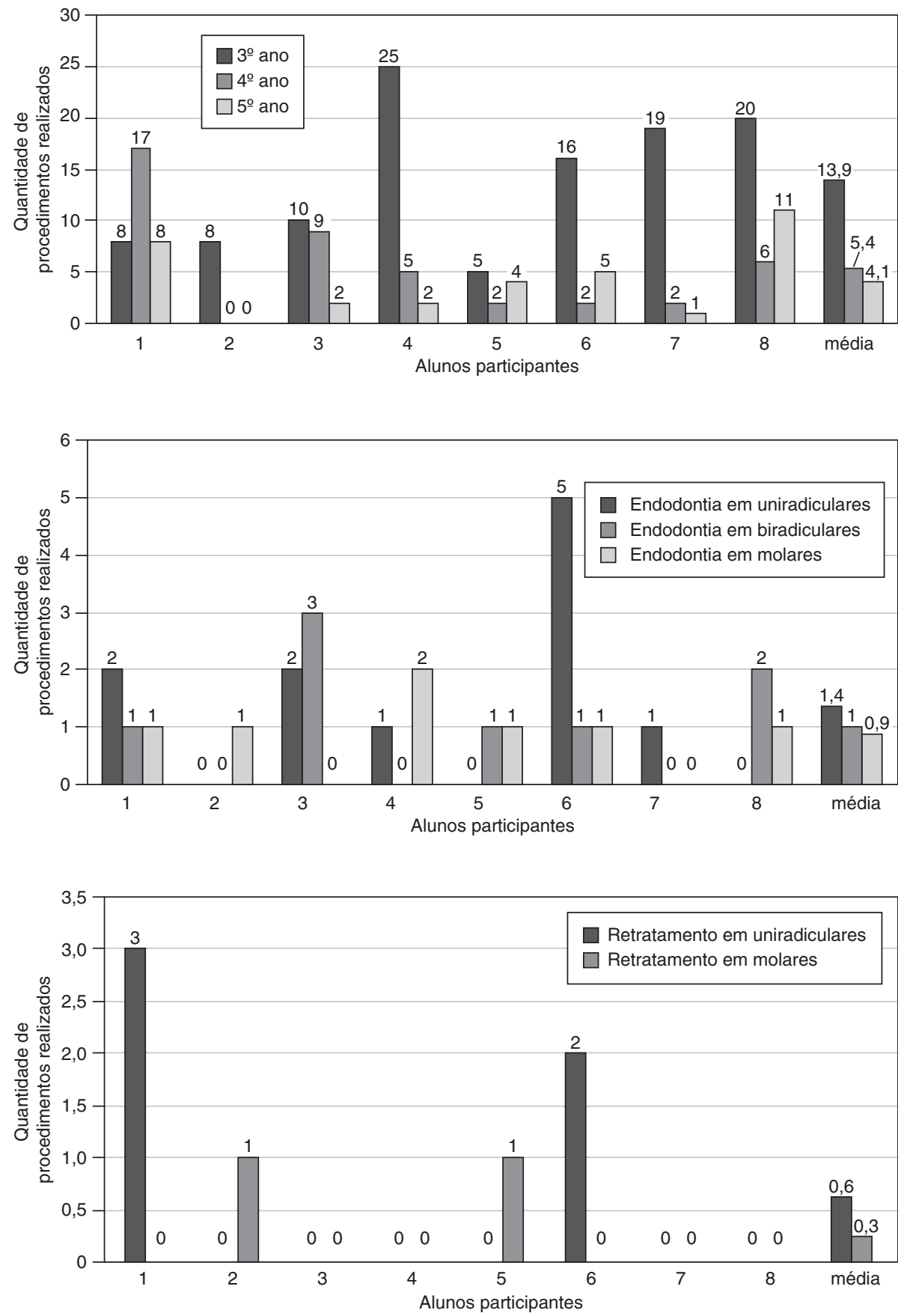

Gráfico 1 - Quantidade de procedimentos periodontais executados na clínica.

Gráfico 2 - Quantidade de procedimentos endodônticos executados na clínica.

Gráfico 3 - Quantidade de retratamentos endodônticos executados na clínica. 
realizaram nenhuma endodontia de biradiculares.

Em relação à produção de endodontia de molares, cuja realização em clínica é escolha opcional dos acadêmicos, a média foi de 0,9 por aluno.

Em relação à retratamentos endodônticos (Gráfico 3), a média foi de 0,87 por aluno. Destaca-se que $50 \%$ dos alunos participantes não realizaram nenhum retratamento endodôntico.

Constatou-se que a produção média foi de 4,1 tratamentos endodônticos por aluno (independente do procedimento realizado). Ressalta-se que a média de consultas necessárias à realização e conclusão dos procedimentos endodônticos foi de 4,8 sessões.

Nos Gráficos 4 e 5 pode-se observar a produção desenvolvida pelos alunos na área de prótese. Ressalta-se que a média de consultas necessárias à realização e conclusão de próteses parciais removíveis e próteses totais foi de 7,9 sessões. Nos procedimentos de prótese fixa, a média de consultas foi entre 4,8 consultas.

A produção média de execução de núcleos metálicos fundidos foi de 1 por aluno enquanto de próteses fixas unitárias foi de 1,6 por aluno (Gráfico 4).
Destaca-se que 37,5\% dos alunos participantes não realizaram pelo menos um desses procedimentos. Ainda na área de prótese fixa destaca-se também que $37,5 \%$ dos participantes não realizaram nenhuma prótese parcial fixa.

$\mathrm{Na}$ área de próteses removíveis (Gráfico 5), todos os alunos realizaram pelo menos uma prótese parcial removível, sendo a produção média de 3,1 por aluno. Já a produção média de execução de próteses totais foi de 1,5 por aluno, sendo que $25 \%$ alunos participantes não realizaram nenhuma prótese total.

Nos Gráficos 6 e 7 pode-se observar a produção desenvolvida pelos alunos na área de dentística.

Em relação aos procedimentos diretos em dentística obtiveram-se os seguintes dados:

- restaurações de amálgama, 2,75 por aluno;

- restaurações de dentes anteriores em resina composta, 13,3 por aluno;

- restaurações de dentes posteriores com resina composta, 12,5 por aluno.

A produção média de restaurações indiretas tipo
Gráfico 4 - Quantidade de procedimentos de prótese fixa executados na clínica.

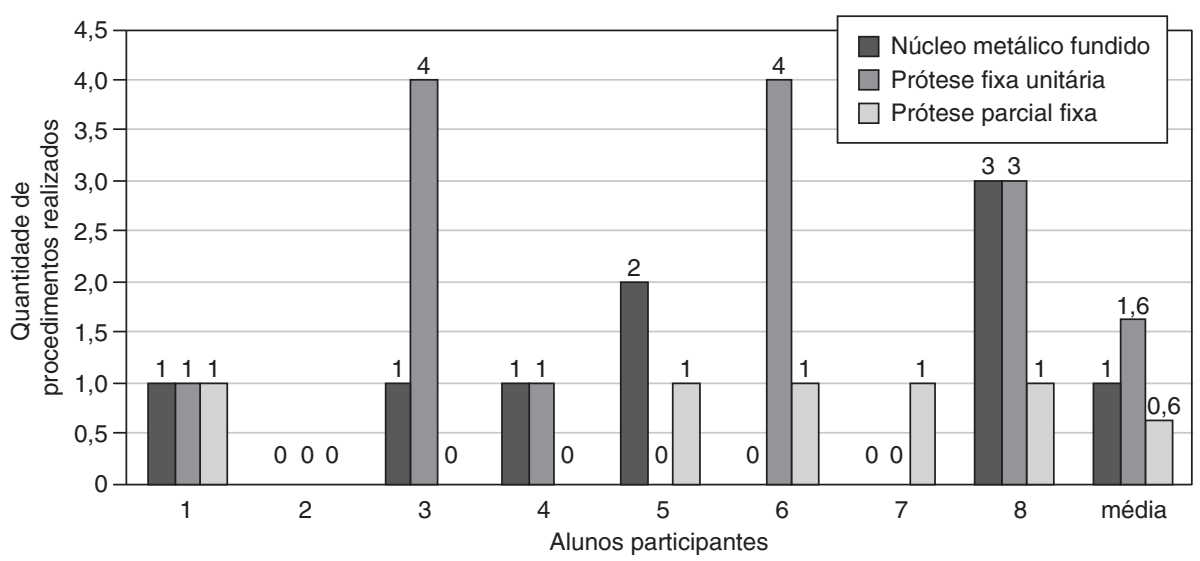

Gráfico 5 - Quantidade de próteses removíveis executadas na clínica.

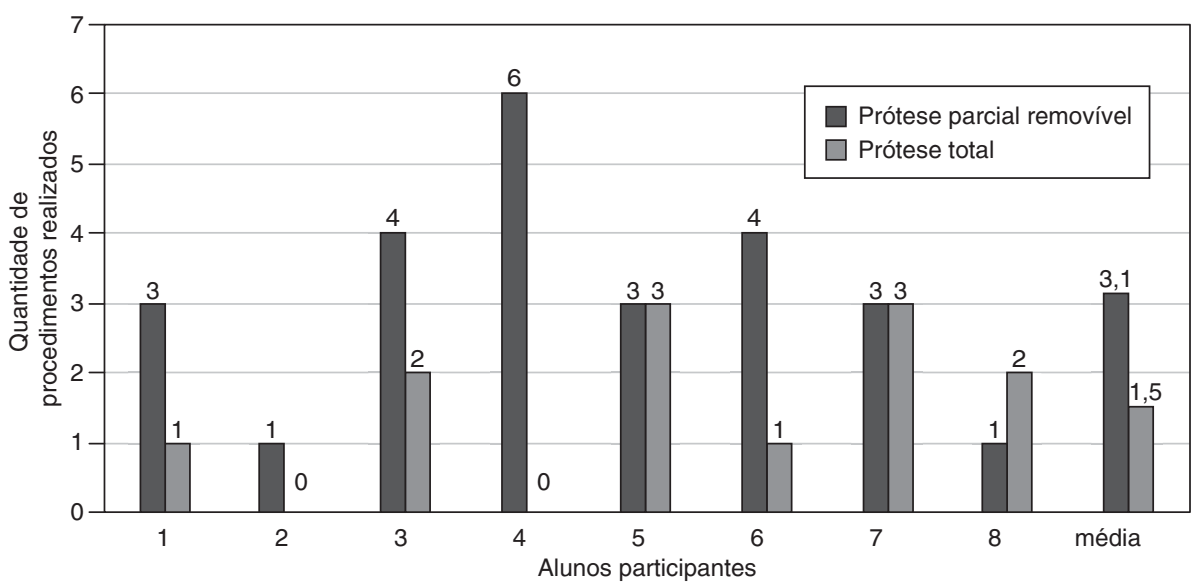



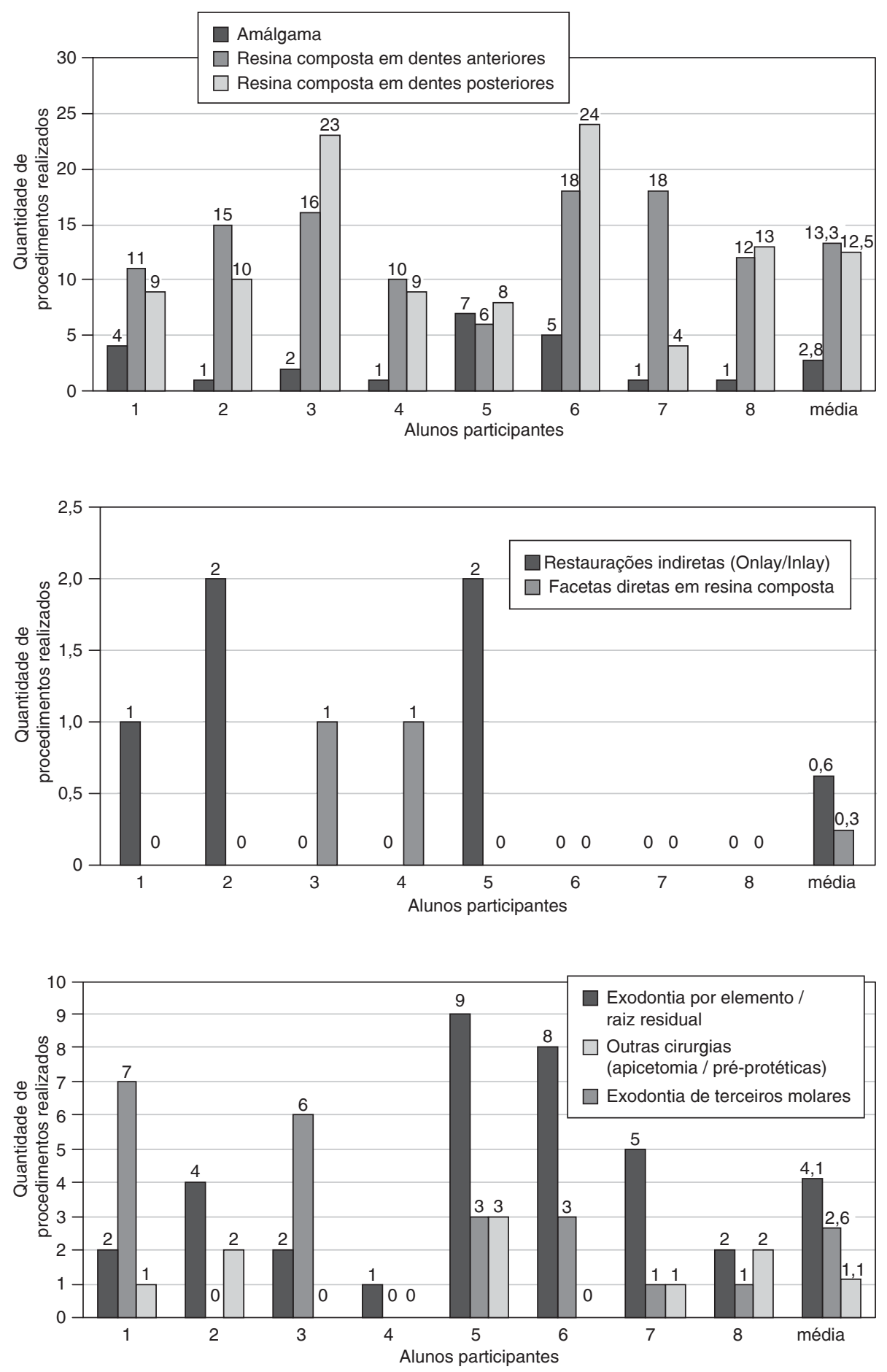

Gráfico 6 - Quantidade de restaurações diretas executadas na clínica.
Gráfico 7 - Quantidade de restaurações indiretas e facetas diretas executadas nas clínicas.
Inlay/Onlay foi de 0,6 por aluno, e de facetas diretas em resina composta, 0,25 por aluno, destacando-se que somente $37,5 \%$ e $25 \%$ dos alunos, respectivamente, conseguiram executar esses procedimentos.

No Gráfico 8 pode-se observar a produção desenvolvida pelos alunos na área de cirurgia nas disciplinas de Atividades Clínicas. São procedimentos executados no centro cirúrgico do curso e integrantes do diagnóstico e planejamento de cada tratamento estabelecidos entre o aluno e seu orientador.

\section{DISCUSSÃo}

Diversos foram os autores que destacaram a importância da clínica integrada na formação do acadêmico em Odontologia. 1,5,7,10,12,15 Especialmente, seu papel na integração do conhecimento entre as 
diversas especialidades com as disciplinas "básicas", visando à adequada elaboração de um plano de tratamento integral do paciente. Outros autores destacaram que a clínica integrada objetiva colaborar na formação do profissional generalista. ${ }^{5,9}$

As atividades clínicas do curso de graduação em odontologia da UNIVILLE foram idealizadas sob as Diretrizes do Conselho Nacional de Educação, na qual os alunos desenvolvem as clínicas de atendimento, desde o início da prática com pacientes, na forma de "clínica integrada" e não por clínicas de especialidades. Este modelo pedagógico permite ao aluno desenvolver uma visão integral do ser humano, propiciando um melhor diagnóstico, planejamento e tratamento dos pacientes. Procura-se respeitar, através do ensino seqüencial por níveis de complexidade, a evolução técnica e científica (teórica) dos alunos, dentro de uma filosofia de promoção de saúde que prioriza uma abordagem multidisciplinar do paciente.

Uma dificuldade, nesse modelo de desenvolvimento da disciplina de atividades clínicas, é o estabelecimento de algum tipo de produtividade mínima, que seria contrário ao Projeto Pedagógico estabelecido pelo curso, que preconiza o atendimento do paciente como um todo, de forma integral, e não por produção técnica. Assim, os acadêmicos executam procedimentos que estão diretamente relacionados ao planejamento dos seus pacientes, que muitas vezes não abrangem todas as especialidades odontológicas.

Com base nos resultados foi possível observar que nesse modelo de desenvolvimento da prática pedagógica em clínica, a produtividade deixa de ser o foco principal e a ênfase incide sobre o desenvolvimento do diagnóstico e planejamento, com uma abordagem integral do paciente.

Os resultados também demonstraram que os acadêmicos podem concluir sua formação sem a execução de procedimentos específicos dentro das especialidades como a realização de uma prótese total, uma prótese fixa ou mesmo uma endodontia. Ao mesmo tempo, constatou-se que alguns alunos apresentaram o desenvolvimento de uma boa produtividade nas diversas disciplinas, sem fugir do foco central do projeto pedagógico do curso - o tratamento integral do paciente.

Um dos aspectos, também observados durante o levantamento de dados da pesquisa, foi a qualidade de informações disponibilizadas nos prontuários. Estas informações devem, dentro de um planejamento de linguagem comum entre todos os discentes e docentes, seguir um padrão de informações que torne possível uma comparação entre os trabalhos executados e quantifica-los. Informações incompletas e deficiência no preenchimento de prontuários dificultam qualquer tipo de levantamento ou pior, ficam sujeitos a interpretações que às vezes não condizem com a realidade executada.

Existe um número de docentes componentes da disciplina de clínica integrada de diferentes especialidades e com variados pontos de vista decorrentes de sua formação. Esta formação especialista pode ocasionar uma deficiência de professores generalistas capazes de instruir os acadêmicos para uma visão global e "generalista", bem como aptos a atuar em todas as áreas da odontologia. ${ }^{5,17}$ No entanto alguns autores consideraram essa diversidade de professores um fator positivo, pois há um aproveitamento das divergências de opiniões entre os docentes a respeito de um mesmo caso clínico e neste momento o aluno fica diante de uma situação real e com isso aprende a respeitar outras opiniões e limites de cada profissional. ${ }^{11}$

Nas clínicas de ensino odontológico das universidades brasileiras foi constatada uma prioridade do processo pedagógico tradicional, ${ }^{2}$ esquecendo-se do paciente e avaliando apenas o aluno de acordo com a competência e produção técnica desenvolvida. Argumentam que, procura-se estimular o aluno no desenvolvimento de atividades curativas que, em muitos casos, são insuficientes na atenção para com a saúde integral de seu paciente.

A formação generalista, humanista, crítica e reflexiva comprometida com o ser humano, respeitando-o e valorizando-o, preconizada pelas DCN, e exercida no modelo pedagógico de clínicas integradas por ora analisadas, nos remete a um novo pensamento em termos de avaliação de desempenho do aluno de odontologia. Estes dados analisados sob a ótica pedagógica que sempre norteou o desenvolvimento dos cursos de odontologia no Brasil no final do século passado podem gerar uma visão distorcida da realidade acadêmica.

As $\mathrm{DCN}^{16}$ também geram conflitos que, quando analisados friamente, desvinculados da realidade do dia a dia do ensino, podem ser mal interpretados. “... atuar multiprofissionalmente, interdisciplinarmente e transdisciplinarmente com extrema produtividade na promoção de saúde..." exige uma visão integral do ser humano, articulado com o contexto social onde existem diferenças entre as pessoas e suas necessidades. Estes contextos de diferenças necessitam de 
uma nova visão de avaliação e ensino. Esta avaliação encontra-se enormemente amparada no binômio diagnóstico e planejamento da realidade encontrada. Não somente da realidade de saúde bucal, mas um diagnóstico e planejamento integral, uma visão do ser humano que se apresenta em dado momento. O conhecimento acumulado para aquele determinado momento deve ser posto a prova de forma que a integração dos conteúdos essenciais do curso de odontologia seja contemplada.

Os dados deste estudo podem gerar uma grande discussão sobre a questão da produtividade dos acadêmicos nas diferentes especialidades odontológicas. No entanto, acreditamos que essa questão contrasta com o modelo de desenvolvimento da disciplina de atividades clínicas previsto nas DCN e o projeto pedagógico do curso que objetiva respeitar o paciente como um ser humano integral, dando ao aluno uma visão generalizada do diagnóstico, planejamento e tratamento do paciente ao invés de uma visão fragmentada da odontologia. Assim sendo, dentro dessa filosofia de promoção de saúde que prioriza o diagnóstico, prevenção e planejamento através de uma abordagem multidisciplinar do paciente, a produtividade individual do aluno deixa de ser o foco principal.

\section{CONCLUSÃo}

De acordo com os dados obtidos, pôde-se concluir que:

1. $\mathrm{Na}$ atual proposta de desenvolvimento da clínica integrada pelas DCN a produtividade individual do acadêmico deixa de ser o foco principal do processo ensino-aprendizagem.

2. O ensino tecnicista abre espaço para uma nova visão na avaliação pedagógica dos acadêmicos.

3. Esta nova visão pedagógica representa a introdução cada vez mais da avaliação qualitativa do desempenho discente.

4. Em relação à produtividade, a realização de outro estudo, com uma amostragem maior, envolvendo um maior número de participantes, e, principalmente, envolvendo escolas com a mesma filosofia de ensino, seria de suma importância para comparação com os resultados obtidos nesse estudo.

\section{ABSTRACT}

Student evaluation and Brazil's National Curriculum Guidelines - The reality of integrated clinics at UNIVILLE

After the publication of the Brazilian National
Curriculum Guidelines (NCG), dentistry schools had to adapt their core curricula. This adaptation not only involves changing the curriculum, but also incorporates a new pedagogic philosophy that seeks to bring NCG precepts closer to the daily teaching practice in the integrated clinics of dental schools. The aim of this study was to analyze the productivity of UNIVILLE's Dentistry School undergraduates in clinical disciplines, for three (3) years of undergraduate study in five (5) different dental specialties (periodontics, operative dentistry, endodontics, prosthodontics, and surgery). The productivity of 8 graduating students was assessed by quantifying their production in three (3) years (third, fourth, and fifth year) of the clinical discipline activities, namely those of low, medium, and high complexity. Data analysis demonstrated the following results: in endodontics, a mean production of 4.1 endodontic treatments per student; in prothodontics, a mean of 1.6 single-unit prosthesis and 1.5 complete denture per student; and, in operative dentistry, a mean of 28 direct restorative procedures per student. Based on the results of this assessment model, it was concluded that the development of clinical activities, both integrated and by level of complexity, allows graduating dental students to acquire effective learning skills, with the follow-up of patients in all the phases of patient diagnosis, as well as treatment planning and conclusion. However, this study demonstrated that students graduated without performing certain technical procedures specific to the researched dental specialties.

\section{DESCRIPTORS}

Integrated Clinics. Productivity. National Curriculum Guidelines. -

\section{REFERÊNCIAS}

1. Almeida RVD, Padilha WWN. Clínica Integrada: é possível promover saúde bucal numa clínica de ensino odontológico? Pesq. Brás. Odont. Clin. Int. 2001a set/dez; 1(3):23-30.

2. Almeida RVD, Gaião L, Padilha WWN. Avaliação do ensino odontológico em clínica integrada. Pesq. Brás. Odont. Clin. Int. 2001 maio/ago; 1(2):29-35.

3. Araújo IC, Araújo MVA, Melo CB, Barroso RFF. Trajetória nacional e internacional do ensino odontológico e a disciplina de clínica integrada nos cursos de odontologia. Rev. Inst. Ciênc. Saúde. 2002 jan/jun; 20(1): 69-73.

4. Arruda WB. Variáveis intercorrentes que influenciam a produtividade clínica no curso de graduação na disciplina de Clínica Integrada(Terapêutica Clínica) da Faculdade de Odon- 
tologia da Universidade de São Paulo. Contribuição ao Estudo. [dissertação]. São Paulo (SP):Faculdade de Odontologia, Universidade de São Paulo/USP;1997.

5. Cardoso DS, Morais ES, Bozzo RO, Oliveira VMB. Análise comparativa do desenvolvimento da clínica em blocos com a da clínica odontológica integrada da UNIARARAS. Revista da ABENO.2006;6(1):35-41.

6. Lombardo I. Reflexões sobre planejamento do ensino de Odontologia. Revista da ABENO.2001;1(1):17-24.

7. Miguel LCM, Ribeiro KMA, Cruz GV, Locks A. Clínica Integrada da UNIVILLE: "a Visão do todo". Revista da ABENO.2006; 6(2):15-108.

8. Milani PAP. Avaliação e produtividade da disciplina de clínica integrada no curso de Odontologia da Universidade Tuiuti do Paraná. [dissertação]. São Paulo (SP): Faculdade de Odontologia, Universidade de São Paulo/USP;2003.

9. Padilha WWN, Medeiros EPG, Tortamano N, Rocha RG. O desenvolvimento da disciplina de clínica integrada nas instituições de ensino odontológico no Brasil.RPG.1995 out/dez; 2(4):193-199.

10. Petroucic F, Rubens FAJ. O ensino na Disciplina de Clínica Integrada. Revista da ABENO.2005; 5 (1):60-64.

11. Poi WR. Clínica Integrada: Do ensino à aprendizagem [tese] São Paulo (SP): Faculdade de Odontologia de São Paulo, Universidade Estadual Paulista;2002

12. Poi WR, Trevisan CL, Lucas LVM, Panzarini SR, Santos CLV. A opinião do cirurgião-dentista sobre a clínica Integrada. Pesq. Brás. Odont. Clin. Int. 2003 jul/dez; 3(2):47-52.

13. Poi WR, Lawall MA, Simonato LE, Gionvanini EG, Panzarini
SR, Pedrini D. Onze anos de avaliação dos planos de tratamento e tratamentos realizados pela disciplina de clínica integrada, Faculdade de Odontologia de Araçatuba - UNESP. Pesq. Brás. Odont. Clin. Int. 2006 set/dez; 6(3):237-242.

14. Reis SMA, Oliveira AG, Magalhães ACP, Santos FG. Verificação da prática interdisciplinar e do nível de integração existente entre os docentes de uma mesma área e de áreas diferentes. Pesq. Brás. Odont. Clin. Int. 2003 jan/jul; 3(1):49-55.

15. Regalin IL, Zaffari F. Produtividade dos acadêmicos nas clínicas do curso de odontologia da UNIVALI [tcc]. Itajaí (SC):Universidade do Vale do Itajaí/UNIVALI; 2007.

16. Resolução CNE/CES3/2002, de 19 de fevereiro de 2002. Institui as diretrizes curriculares nacionais dos cursos de graduação em Odontologia. Diário Oficial da União. Séc. 1, p.10, Brasília, 04 mar 2002. (Conselho Nacional de Educação. Câmara de Educação superior).

17. Rodrigues MM, Reis SMAS. A Interdisciplinaridade e a Integração no Ensino Odontológico: Reflexos sobre o Perfil Profissional em Relação às Reais Demandas na Maioria da População por Atenção Odontológica. Extensão.2004 set; 4(1): 21-27.

18. Romero SS, Vasquez AMM, Puertas KV, Simone JL. Produtividade clínica dos alunos do curso de graduação da disciplina de clínica odontológica integrada da Faculdade de Odontologia da Universidade de Santo Amaro. Rev. Odontol. Univ. Santo Amaro. 2003 jan/jul; 8(1): 4-10.

Recebido em 08/10/2012 Aceito em 10/12/2012 